\title{
Syntactic complexity and elaborative processing
}

\author{
MARK A. MCDANIEL \\ University of Colorado, Boulder, Colorado 80302
}

\begin{abstract}
Memory for sentences as a function of the syntactic complexity of the sentences was examined. Sentence complexity was varied through a manipulation that involved presenting sentences in either self-embedded forms or more standard forms. Subjects performed an incidental semantic orienting task on a set of sentences varying in complexity and were subsequently tested for their recognition memory of the sentences. In Experiment 1, subjects were tested for their memory of both surface characteristics and meaning of the sentences. There were no differences caused by sentence complexity for memory for meaning. Memory for surface structure, however, was a function of sentence complexity such that there was better memory for the more complex sentences. Experiment 2 replicated the finding that the more complex sentences produced better recognition memory for surface structure. The results are interpreted within a framework that suggests that increased syntactic complexity produces more elaboration, which in turn produces better memory.
\end{abstract}

In recent years, much interest has centered around a levels-of-processing approach to memory (Craik \& Lockhart, 1972; Lockhart, Craik, \& Jacoby, 1976). This approach assumes that when a stimulus is presented or an event occurs, it is analyzed by pattern recognition and other cognitive processes. Memory is viewed as a by-product of these cognitive operations. How well something will be remembered is a function of how "deeply" it is processed.

Initial conceptualizations of this approach (Craik \& Lockhart, 1972) associated greater depth with a greater degree of semantic processing. More recently, however, Lockhart et al. (1976) have suggested that depth may refer to two somewhat different aspects of processing. In addition to the original notion of depth, Lockhart et al. discuss depth in terms of the further analysis or elaboration of a stimulus within one particular qualitative domain (e.g., phonemic, syntactic, or semantic). This formulation, then, views good memory not only as a function of the nature of an event's features that are analyzed, but also as a function of the number of features that are analyzed.

Experiment 1 was performed as part of a master's thesis submitted to the University of Colorado. The author is indebted to Gregory A. Kimble, Richard K. Olson, and the late William F. Battig for their helpful suggestions throughout the course of this project. Gratitude is also extended to Donald J. Foss for making his materials available for use, to Susan Stahl for help in scoring the data, and to Michael E. J. Masson and Linda S. Sala for comments on an earlier draft of this paper. The results of Experiment 1 were reported in part at the meetings of the American Educational Research Association in San Francisco, April 1979. This research was supported in part by the National Science Foundation Grant BNS 72-02084. Requests for reprints should be addressed to Mark McDaniel, who is now at the Department of Psychology, University of Notre Dame, Notre Dame, Indiana 46556.
Lockhart et al. (1976) additionally hypothesize that the operations carried out on a stimulus (i.e., the nature and number of features analyzed) will depend on, among other things, the materials that are to be processed. Specifically, Lockhart et al. maintain that if the material is uncommon, unfamiliar, or difficult to process, then many features will be analyzed, and this will result in a well remembered, rich memory trace. Further explicating their views, Jacoby and Craik (1979) develop the idea that the beneficial effects of more elaborate encodings are due to the dimension of distinctiveness; items that are difficult to encode will generally result in good memory because "difficulty necessitates more extensive processing, which then results in the formation of a more distinctive trace" (p. 12). The importance of this formulation follows: "Since this distinctiveness is relative to a particular context or encoding dimension, this dimension must be reinstated at retrieval (for the distinctness of the trace to be effectively utilized)" (Jacoby \& Craik, 1979, p. 19). In a similar vein, Bransford, Franks, Morris, and Stein (1979) have suggested that the value of elaborative processing will be constrained by the retrieval situation. Theoretically, then, the influence of material difficulty on memory should also depend on the retrieval situation.

While much experimentation concerning levels of processing exists, there have been few studies that have directly investigated the effects of materials-in particular, material unfamiliarity or difficulty-on encoding and memory. One set of experiments manipulated sentence difficulty at a perceptual level (Kolers, 1973, 1976; Kolers \& Ostry, 1974; Masson \& Sala, 1978). Typically, sentences were presented in one of two typographies: a normal typography and a transformed typography (upside down or backward). The results from these experiments showed that sentences 
that were originally presented in transformed typography were better recognized than sentences that were presented in normal typography.

Kolers' $(1973,1976)$ interpretation of this memory effect is that the inverted typographical sentences required additional encoding operations, leading to good memory for the graphemic phase of analysis. At this early perceptual level of sentential processing, then, there is some support for the Lockhart et al. (1976) ideas.

The following experiments were conducted to investigate the generality of these ideas. Unlike previous work, sentences were made more or less complicated at a syntactic level. Further, the retrieval tasks were varied to examine how material difficulty might differentially affect memory performance due to differences in the test task.

\section{EXPERIMENT 1}

The difficulty or complexity of processing the material was manipulated by changing the syntactic form of the sentences. The complex sentences were self-embedded sentences, like "The car that the man whom the dog bit drove crashed." The easier sentences were of this syntactic form: "The car driven by the man that the dog bit crashed."

The use of this kind of material was chosen for two reasons. First, there was evidence supporting the intuition that the difficulty manipulation was valid, that it did influence the difficulty of processing. Previous research had shown that, in general, self-embedded sentences with relative pronouns deleted are harder to comprehend than self-embedded sentences with relative pronouns included (Fodor \& Garrett, 1967; Foss \& Lynch, 1969; Hakes \& Cairns, 1970; Hakes \& Foss, 1970). As a preliminary investigation to the present work, these findings were corroborated and extended to include the more regular sentence forms. Twentyfive students from an introductory psychology course at the University of Colorado rated the three forms (self-embedded with relative pronouns deleted, selfembedded with relative pronouns included, and "regular") for each of 30 sentences for ease of comprehensibility. The self-embedded forms were rated as being more difficult to process than the regular forms (see Table 1 for the mean ratings for each sentence), and, with only a few exceptions, the self-embedded forms with relative pronouns deleted were rated as being more difficult to process than the self-embedded forms with relative pronouns included. Thus, with this syntactic manipulation, three levels of processing difficulty or complexity were obtained.

A second reason for choosing this kind of material was that the syntactic manipulation did not affect any other obvious dimension of the sentences that might also influence memory, such as meaning, the particular
Table 1

Mean Difficulty Ratings for Each Sentential Form of Each Sentence

\begin{tabular}{|c|c|c|c|}
\hline \multirow{3}{*}{$\begin{array}{l}\text { Sentence } \\
\text { Number }\end{array}$} & \multicolumn{3}{|c|}{ Sentence Form } \\
\hline & \multirow[b]{2}{*}{ Regular } & \multicolumn{2}{|c|}{ Self-Embedded } \\
\hline & & Unreduced & Reduced \\
\hline 1 & $4.20^{*}$ & 7.20 & 8.00 \\
\hline 2 & $2.20 *$ & 6.00 & 6.20 \\
\hline 3 & 6.20 & 6.00 & 7.10 \\
\hline 4 & $2.30^{*}$ & $6.20^{*}$ & 7.50 \\
\hline 5 & $2.40^{*}$ & $5.20^{*}$ & 7.30 \\
\hline 6 & $4.40^{*}$ & 7.90 & 7.80 \\
\hline 7 & $1.90^{*}$ & 6.80 & 7.50 \\
\hline 8 & $4.80^{*}$ & $5.90^{*}$ & 7.80 \\
\hline 9 & $2.20^{*}$ & $5.30^{*}$ & 6.40 \\
\hline 10 & $1.80^{*}$ & 5.60 & 6.30 \\
\hline 11 & $3.30^{*}$ & $5.60^{*}$ & 7.80 \\
\hline 12 & $2.30 *$ & 7.20 & 7.00 \\
\hline 13 & $4.20^{*}$ & $6.50 *$ & 7.30 \\
\hline 14 & $3.40^{*}$ & 6.60 & 7.20 \\
\hline 15 & $3.20^{*}$ & $6.10^{*}$ & 7.20 \\
\hline 16 & $3.20 *$ & $4.90^{*}$ & 7.20 \\
\hline 17 & $3.00^{*}$ & 6.20 & 6.60 \\
\hline 18 & $3.80^{*}$ & $5.60^{*}$ & 6.90 \\
\hline 19 & $3.60^{*}$ & $6.00 *$ & 7.40 \\
\hline 20 & $1.90^{*}$ & $6.00 *$ & 6.90 \\
\hline 21 & $2.70^{*}$ & $6.40^{*}$ & 7.30 \\
\hline 22 & $2.40^{*}$ & 6.80 & 6.90 \\
\hline 23 & $3.20 *$ & $5.70^{*}$ & 7.10 \\
\hline 24 & $2.60^{*}$ & $5.40^{*}$ & 7.20 \\
\hline 25 & $2.30 *$ & $5.00 *$ & 7.20 \\
\hline 26 & $1.70^{*}$ & $5.40 *$ & 7.00 \\
\hline 27 & $2.80^{*}$ & $5.30^{*}$ & 7.00 \\
\hline 28 & $2.40^{*}$ & $5.60^{*}$ & 6.60 \\
\hline 29 & $4.20^{*}$ & $6.40^{*}$ & 7.40 \\
\hline 30 & $2.70^{*}$ & $5.50^{*}$ & 7.00 \\
\hline Overall & 3.01 & 6.01 & 7.14 \\
\hline
\end{tabular}

Note-Lower ratings represent easier comprehensibility. $\quad *$ Significantly different from the entry in the column that immediately follows (by sign test).

lexical items used, and sentence length. A potential source of confounding was therefore avoided. It might still be argued, though, that the doubly embedded sentences could not be comprehended as accurately as the normal sentences. If this were the case, then level of comprehension, and not level of difficulty, might be responsible for any retention differences observed (Bransford \& McCarrell, 1974). While this could not be ruled out a priori, the observed pattern of results suggested that this was not the case.

The present experiment tested for memory of the sentences in two ways. In one test, subjects were required to recognize paraphrases of the sentences. Performance on this test depended on remembering the meaning of the sentences. In the other test, subjects were presented the sentences in each syntactic form and were required to select the syntactic form in which the sentence had originally been presented.

These tests provided an interesting context within 
which to test the levels-of-processing notions outlined above. It will be recalled that according to Lockhart et al. (1976), the sentences that are more difficult to process (the embedded forms) should induce more elaboration and, as a by-product of that elaboration, produce traces containing more information about those sentences. Moreover, since more information about the complex sentences is encoded, those sentences should be better remembered than the easier sentences; embedded forms should produce higher scores on the recognition tests than do more regular forms.

Jacoby and Craik (1979), however, stress consideration of the retrieval environment. They theorize that the distinctiveness resulting from elaboration will enhance recognition only if the distinctive encoding dimension is reinstated at retrieval. If it is assumed that the elaborated, distinctive dimension of the encoding will be associated with syntactic information, then the embedded forms should be better recognized than the regular forms on the test for syntactic form, but not necessarily on the test for meaning.

Finally, assuming that the syntactically more complex sentences take longer to process than the less complex sentences, a total-time hypothesis (Cooper \& Pantle, 1967) would predict that the more complex sentences should be remembered better than the easier sentences. Since this prediction is the same as that motivated by the Lockhart et al. (1976) framework, how can one distinguish between the two? One strategy would be to manipulate the amount of time that the sentences are presented for processing and then compare the effects of presentation rate on memory performance with the effects of sentence complexity. If the pattern of results for sentence complexity and for presentation rate were different, it would imply that the effects of complexity were not due to amount of time per se.

\section{Method}

Materials and Design. The following three sentence types were chosen for use. (1) The car the man the dog bit drove crashed. (2) The car that the man whom the dog bit drove crashed. (3) The car driven by the man that the dog bit crashed.

Sentence Type 1 is self-embedded with relative pronouns deleted (self-embedded reduced), Sentence Type 2 is selfembedded with relative pronouns included (self-embedded unreduced), and Sentence Type 3 is of more familiar construction, referred to here as "regular." Thirty sentences were selected from the Foss and Lynch (1969) materials and presented in one of these three forms. (These were the same sentences for which the ease of comprehension ratings were obtained.) Processing difficulty was a within-subjects variable so that each subject saw one-third of the 30 sentences in each form. The particular form in which a sentence was presented was counterbalanced across subjects. Interspersed among the 30 target sentences were 10 filler sentences included in an attempt to prevent subjects from developing a more automatic processing strategy for the selfembedded sentences than would ordinarily be the case. All sentences were typed on $35-\mathrm{mm}$ slides for presentation purposes.

Two presentation rates were used for displaying the sentences to the subjects, a $10-\mathrm{sec}$ and a 15 -sec rate. Presentation rate was manipulated as a within-subjects variable and was counterbalanced so that each form of every sentence was displayed with both presentation rates across subjects. Pilot work indicated that $10 \mathrm{sec}$ approximated a lower limit for enabling subjects to process the sentences. The $15 \mathrm{sec}$ rate provided a rate in which subjects could process the sentences comfortably, but with little time to spare.

For the retention phase of the experiment, two types of memory tests were constructed, both of which utilized threealternative forced-choice formats. One test was designed to measure how much syntactic structure was remembered. This test contained 30 questions. A question was composed of a sentence from the target set typed in all three of its possible forms. The other test was designed to measure the amount of meaning retained. There were 60 items on this test, 2 items derived from each of the 30 target sentences. Each sentence yielded two items by virtue of the fact that memory for the meaning of the main clause (the outermost clause of a sentence) was tested and memory for the meaning of one of the subordinate clauses (an inner clause) was tested. For a particular test item, the three choices were constructed in the following manner. The clause being tested was paraphrased such that the word representing either the object or the subject of the phrase remained unchanged and the remaining lexical items were changed in such a way as to preserve the meaning. Two distractor items were constructed that used the same unchanged word but in a sentence that did not preserve the meaning of the clause being tested. For example, one test question for the sentence "The disease that the medicine which the scientist discovered cured damaged the liver" was: (a) the disease harmed the organ; (b) the disease weakened the joint; (c) the disease discolored the skin. As can be seen from the example, an attempt was made to construct the distractors such that their meaning would be compatible with the context of the sentence being tested. It was decided to leave unchanged one of the words from the actual sentence in order to more clearly identify the sentence as a whole, since some of the sentences had overlapping themes (e.g., teacher-student, child-mother).

In constructing the tests, care was taken to counterbalance several factors. First, the position of the alternatives was varied so that the correct alternative appeared equally often in each position (i.e., first, second, and third). Second, for the gist test, half of the questions testing memory for the main clause preceded the corresponding questions for the subordinate clause and vice versa. Third, two forms of the gist test were constructed, one form being identical to the other except that the order of the two test questions for each sentence was reversed. This was done to control for possible cuing effect of one test question on the other for each sentence.

The last experimental variable, test order, concemed the order in which the two types of tests (surface and gist) were administered. This was primarily of methodological interest. Intuitively, it would seem that the test for meaning would not contaminate the results of a subsequently presented test for memory of syntactic structure. It was felt that, if this were true, it would be at least comforting to demonstrate it. On the other hand, if it were not true, some interesting questions might be raised. A similar kind of rationale could be presented for examining the parallel question of whether the test for syntactic structure influences performance on the test for meaning.

Procedure. Subjects participated in groups of one to four. They were instructed that they would be performing a comprehension task. Briefly, they were told that they would be viewing phrases (the term "phrases" was used since some subjects objected that the self-embedded forms were not grammatically correct sentences) that they should read and try to understand. After reading each phrase, they would be given a test (a wordchoice orienting task) to see if they had been able to understand each phrase. During the course of the instructions, five examples were presented. The example set of sentences consisted of a regular sentence, a filler sentence, a self-embedded unreduced sentence, a self-embedded reduced sentence, and a self-embedded unreduced sentence, in that order. The example set was pre- 
sented twice: the first time, the examples were subject paced, with the experimenter offering aid in interpreting the embedded forms; the set was then presented a second time in order to familiarize subjects with the presentation rates used in the experiment. Finally, the instructions stressed that it was important to think about the sentence for the entire time it was being presented, since the comprehension test was not easy.

The set of 40 sentences ( 30 target items and 10 fillers) was next presented, using the following procedure. A sentence was projected on a blank wall in front of the subjects by a Carousel slide projector. After the appropriate time interval (either 10 or $15 \mathrm{sec}$ ), the projector advanced to the next slide, which contained a word pair. The word pair consisted of one word that was congruent or consistent with the meaning of the sentence and another word that was inconsistent with the meaning of the sentence. Approximately equal numbers of the key members of the word pairs were related to the different clauses of the sentences. Therefore, there was no systematic sentential location that cued the correct answer, eliminating any reason for selective processing of certain parts of the sentences. Subjects attempted to choose the word that was the most semantically related to the sentence they had just viewed, and they recorded their responses on an answer sheet. The word pairs were presented for $6 \mathrm{sec}$, at the end of which a new trial began.

Upon completing the sentence set, subjects were given an interpolated task, because pilot work had indicated that an immediate recognition test would be subject to ceiling effects. The task was part of another totally unrelated experiment. It involved having subjects assess the validity, with regard to themselves, of a set of statements describing personality attributes. Subjects were told that they would later be tested for their memory of how they responded to each statement. The statements were printed on a sheet of paper, and subjects marked "true" or "false" beside each statement, depending on whether or not they thought the statement described them. It took approximately $10 \mathrm{~min}$ for subjects to respond to the statements, after which they were given the recognition tests for the sentences presented in the first part of the experiment. Each recognition test was administered separately, the second being given only after the entire group had finished the first. Subjects were instructed to make a best guess if they were not sure of an answer. When the subjects were finished with the second recognition test, they were asked to indicate on the back of the test if they had expected to be tested for their memory of the sentences. The subjects who replied affirmatively were replaced. The experimental session ended with subjects completing the interpolated experiment described above.

Subjects. Subjects were 48 introductory psychology students at the University of Colorado participating in partial fulfillment of a course requirement. One half of the subjects were given a meaning recognition test first, followed by a syntactic structure recognition test. The other half were given the syntactic structure recognition test first, followed by the meaning recognition test.

\section{Results}

Comprehension. It was of interest to determine whether the self-embedded sentences were comprehended as well as the regular sentences. If not, effects of sentence type on memory could be explained by differential comprehension of the sentence types. That is, any differences in memory between the sentence types could be explained by positing either that the different sentence types were interpreted differently or that subjects were simply not able to extract enough meaning from the self-embedded sentences. One indication of whether subjects extracted meaning equally well from the different sentence forms is how they responded on the word-choice task.

The response protocols from the word-choice task were scored for the proportion of errors made. In general, no more errors were committed on the selfembedded forms than on the regular forms. In fact, more errors were made, on the average, when the sentences were in regular form (6.7\%) than when they were in either self-embedded unreduced form $(4.3 \%)$ or selfembedded reduced form (4.3\%). These data were analyzed with a two-way within-subjects analysis of variance. The within-subjects variables were sentence type (regular, self-embedded unreduced, and self-embedded reduced) and presentation rate (10 and $15 \mathrm{sec})$. The only reliable effect was that of sentence type $[F(2,94)=3.73$, $\mathrm{MSe}=.005]$. Even though the sentence type effect was reliable, the observed difference between the sentence types was so small (the average difference between the regular and self-embedded types was .2 errors out of a possible 10 ) that the practical significance of the effect is questionable. Further, the fact that the absolute number of errors made was low $(5.1 \%$, on the average) seems to indicate that the sentences were, in general, comprehended (as assessed by orienting mistakes). Thus, according to the results of the word-choice test, sentences were well comprehended regardless of the presentation rate and sentence form, although there was a slight but significant difference in number of orienting mistakes as a function of sentence form.

Retention. The mean proportion of items recognized on each test as a function of sentence form, presentation rate, and test presentation order is shown in Table 2 . From the table, it can be seen that sentence form, in general, had little effect on recognition levels. In fact, it appears that the only factor that produced consistent differences was the type of memory being tested, with

Table 2

Mean Proportion of Items Recognized as a Function of Type of Test, Test Presentation Order, Sentence Form, and Presentation Rate

\begin{tabular}{|c|c|c|c|c|c|}
\hline \multirow[b]{3}{*}{ Sentence Form } & \multicolumn{5}{|c|}{ Presentation Rate } \\
\hline & \multicolumn{2}{|c|}{$\begin{array}{l}\text { Surface-First } \\
\text { Test Order }\end{array}$} & \multicolumn{2}{|c|}{$\begin{array}{l}\text { Gist-First } \\
\text { Test Order }\end{array}$} & \multirow[b]{2}{*}{ Mean } \\
\hline & 10 & 15 & 10 & 15 & \\
\hline & \multicolumn{5}{|c|}{ Surface Test } \\
\hline Regular & .42 & .42 & .48 & .58 & .47 \\
\hline Self-Embedded Unreduced & .45 & .37 & .40 & .48 & .42 \\
\hline \multirow[t]{2}{*}{ Self-Embedded Reduced } & .52 & .59 & .45 & .42 & .49 \\
\hline & \multicolumn{5}{|c|}{ Gist Test } \\
\hline Regular & .83 & .86 & .77 & .78 & .81 \\
\hline Self-Embedded Unreduced & .79 & .88 & .68 & .76 & .78 \\
\hline Self-Embedded Reduced & .87 & .84 & .76 & .79 & .81 \\
\hline
\end{tabular}

Note-Presentation rate $=$ number of seconds the sentence was displayed. 
memory for meaning being better than memory for surface features. These impressions were confirmed with a four-factor mixed analysis of variance with three within-subjects variables (sentence form, presentation rate, and test type) and one between-subjects variable (test presentation order). There was no main effect of sentence form, and recognition for meaning was better than recognition for surface information $[F(1,46)=$ 276.27, $\mathrm{MSe}=.06]$.

In addition to the main effect of test type, the analysis of variance produced two significant interactions. Test type interacted with test order such that the level of recognition for the surface structure of the sentences was the same regardless of whether it was tested after (.46) or before (.46) memory for meaning, whereas levels of recognition for meaning were higher when the meaning test was administered after the surface test $(.84)$ than when it was administered before the surface test $(.76)[\mathrm{F}(1,24)=6.53, \mathrm{MSe}=.05]$. There was also a significant four-way interaction among test type, sentence type, presentation rate, and test order $[\mathrm{F}(2,48)=4.85, \mathrm{MSe}=.02]$. One way to interpret this interaction is in terms of how different variables affected surface memory compared with how they affected memory for meaning. Memory for meaning was better when the gist test was administered after the surface test. Also, presenting the sentence for $15 \mathrm{sec}$ produced better recognition for meaning than did presenting the sentences for $10 \mathrm{sec}$. Sentence type had almost no influence on memory for meaning. By contrast, recognition for surface memory was not influenced in a straightforward manner by either test order or presentation time. Rather, the amount of memory for surface features was influenced by a complex interaction among sentence type, presentation rate, and the order of test administration.

Separate analyses of variance for the meaning and surface recognition scores supported the kind of interpretation discussed above. The analysis for the meaning test showed that memory was better when the meaning test was administered last rather than first $[F(1,46)=$ 7.01, $\mathrm{MSe}=.08]$ and that recognition was also better when the sentences were presented for $15 \mathrm{sec}$ compared with $10 \mathrm{sec}[\mathrm{F}(1,46)=4.26, \mathrm{MSe}=.02]$. No other effects were reliable. On the other hand, the analysis for the surface test indicated no reliable effects; the three-way interaction among sentence type, presentation rate, and test order was marginally significant $[F(2,92)=$ $2.82, \mathrm{p}<.07]$, however.

Thus, it appears that sentence form, the variable of major interest, has no effect on how well the meaning of the sentence is remembered and has a complex effect, if any, on memory for the surface structure. The particular pattern of results for the surface test with regard to sentence type may be misleading, however. The recognition test, as it was scored, may have yielded a measure of memory for the regular form of the sentences that is incomparable with the measures of memory for self- embedded unreduced and self-embedded reduced sentences. To correctly identify a regular sentence, the subject only had to remember the kind of syntactic information that distinguishes a regular form from a self-embedded form. But to correctly identify either a self-embedded unreduced or self-embedded reduced sentence, the subject had to remember not only syntactic information, but also the precise lexical information that distinguishes a self-embedded unreduced form from a self-embedded reduced form (absence or presence of relative pronouns). In other words, correct recognition of a self-embedded unreduced or self-embedded reduced form required more information to be remembered than correct recognition of a regular sentence. To make the recognition data more comparable, the recognition tests were rescored so that, for self-embedded reduced and self-embedded unreduced sentences, an answer only had to indicate that it was a self-embedded form to be correct. The resulting data may be viewed as a measure of how well subjects could remember the syntactic form of the sentences.

These data had to be transformed for the purpose of analysis because not only did the number of possible hits differ for regular vs. self-embedded sentences, but also the probability of a hit, given no memory, differed (33\% for regular sentences and $67 \%$ for self-embedded sentences). Therefore, proportions were calculated representing the actual number of items correctly recognized minus the number of items expected to be recognized by chance divided by the number representing perfect responding above that expected by chance.

A one between- and two within-subjects analysis of variance was performed on these derived scores, with the between-subjects variable being test order and the within-subjects variables being sentence type and presentation rate. The main effect of sentence type was the only reliable effect $[F(1,46)=10.31$, $\mathrm{MSe}=.47]$. When the sentences were presented in selfembedded form, surface structure was better remembered than when the sentences were presented in the regular form (.53 of the total possible above chance for the self-embedded forms compared with .21 of the total possible above chance for the regular forms). From Table 3, it can be seen that the difference in recognition levels between the self-embedded and regular forms was greater when the surface test was administered before, rather than after, the meaning test. However, this interaction was only marginally significant $[F(1,46)=3.81$, $\mathrm{p}<.06]$. Additional analyses were carried out to determine if the observed levels of recognition were greater than chance performance. Recognition of surface structure was significantly above chance for both selfembedded and regular forms $[\mathrm{F}(1,95)=90.76, \mathrm{MSe}=$ .29 , and $\mathrm{F}(1,95)=24.68$, MSe $=.17$, respectively]

A further analysis was carried out on the scores for the meaning test to investigate how well main clauses were remembered compared with subordinate 
Table 3

Mean Proportion of Items Recognized Above Chance on the Surface Memory Test as a Function of Sentence Form and Test Order

\begin{tabular}{lcc} 
& \multicolumn{2}{c}{ Test Order } \\
\cline { 2 - 3 } Sentence Form & Surface First & Gist First \\
\hline Regular & .13 & .29 \\
Self-Embedded & .64 & .41 \\
\hline
\end{tabular}

Note-The proportions given indicate the number of items recognized above chance divided by the upper limit of the number of items that could be recognized above chance.

clauses and to see whether sentence form interacted with any differences between clause type. On the average, more main clauses were remembered than subordinate clauses $(.84$ and .76 were the respective mean proportions). The results of a four-factor mixed analysis of variance, with clause type, sentence type, presentation rate, and test order as the factors, indicated that the superior memory performance for the main clauses was reliable $[\mathrm{F}(1,46)=35.72, \mathrm{MSe}=.03]$. The only other reliable effect from the analysis was test order, with better performance occurring when the test was given after the surface test rather than before it $[\mathrm{F}(1,46)=7.03, \mathrm{MSe}=.17]$. There were no sentence type effects, indicating that the form in which the sentence was presented did not differentially affect memory for the two clause types.

In summary, the results of major importance related to whether presenting a sentence in different forms would influence memory for that sentence. The results showed that memory for the meaning of a sentence was not influenced by the form in which the sentence was presented. Memory for the surface structure of a sentence, however, differed, depending on the syntactic form in which the sentence was presented when scores reflecting a discrimination between the two major syntactic arrangements (regular and self-embedded) were considered. Specifically, the more difficult sentences to process (self-embedded forms) were identified correctly more often than the sentences more easily processed (regular forms), regardless of presentation rate, when the test was administered, and how memorable the sentence was in general.

\section{Discussion}

These results appear to be consistent with the ideas proposed by Jacoby and Craik (1979) and Lockhart et al. (1976). They claim that a stimulus that is difficult to process will require more extensive cognitive analysis and thus produce a distinctive trace; a highly practiced stimulus, on the other hand, will be analyzed by fewer operations, and an impoverished, less distinguishable memory trace will result. These predictions were confirmed, in that the more complex sentential material was remembered as well for meaning and better for syntactic form than was the easier sentential material.
Moreover, this pattern supports an explication of the effect of stimulus difficulty on memory that is more precise than that originally proposed by Lockhart et al. (1976). As Jacoby and Craik (1979) note, the kind of memory performance supported by a distinctive trace will depend on the retrieval conditions. For good memory performance, the particular context or encoding dimension for which the trace is distinctive must be be reinstated at retrieval. Indeed, only on the test that provided information about the syntactic dimension, the dimension on which the self-embedded sentences were more complex or difficult, did the self-embedded sentences demonstrate better memory than the regular sentences. On the meaning test, when there were no cues to reinstate the dimension on which difficulty differed, memory performance was comparable for all sentence types. Phrased differently, it could be said that the richer trace resulting from the more extensive processing required by difficult material is richer in terms of the information specific to the domains for which further processing is necessary. It is only the information from these domains, and not other kinds of information, for which we should find better memory for difficult material.

This kind of explanation is similar to a transferappropriate processing notion (Bransford et al., 1979; McDaniel, Friedman, \& Bourne, 1978) that holds that whether or not a particular type of processing will produce good memory performance depends on the kind of information required by the test environment. In the context of the present study, the implication would be that more extensive syntactic processing was induced by the self-embedded sentences. This more extensive processing was beneficial for memory performance, but only when the test required the use of that type of information.

It could be argued that the syntactic complexity of the self-embedded sentences induced further elaboration in the semantic domain, as well as the syntactic domain. If so, following the logic above, one might wonder why self-embedded sentences were not also better remembered for meaning. Even if syntactic complexity did induce more elaboration in a semantic domain, however, the demands of the orienting task required that semantic information be elaborated, so that it would be expected that all sentences, regardless of type, were well processed semantically. Hence, in view of the nature of the orienting task, equal memory for meaning between the sentence types is not contradictory to the position outlined above, even if syntactic complexity did induce processing in the semantic domain.

A more parsimonious, although perhaps less theoretically interesting, explanation of the effect of processing difficulty on memory could be offered. It may be that the more difficult sentences were better remembered because subjects spent more time on them. Such a finding would be consistent with the total-time view men- 
tioned in the introduction (Cooper \& Pantle, 1967). This appears not to be the case, however, because when subjects were given more time to examine the sentences (15-sec presentation rate), they remembered more of the meaning of the sentences but not more of the surface form. This indicates that the superior memory for surface form produced by the more complex sentence types was not a result of differences in processing time between self-embedded and regular sentences.

One might still offer the objection that the higher memory scores for surface form for the self-embedded sentences were simply an artifact of the post hoc scoring procedures used. Clearly, a more straightforward comparison of recognition performance (for surface form) for self-embedded vs. regular sentences would be desirable.

\section{EXPERIMENT 2}

Experiment 2 was performed to provide a direct comparison between the two sentence types (regular and self-embedded) for memory of surface structure. In this experiment, memory was tested with a twoalternative forced-choice test, in which one alternative was the sentence in regular form and the other alternative was the sentence in self-embedded unreduced form. If the memory differences found in Experiment 1 were an artifact of the scoring procedure, we would not expect to find such differences in the following experiment. On the other hand, if the differences found in the first experiment do reflect differences in memory levels, then we would expect the same pattern of results (for memory of surface information) to emerge again.

\section{Method}

Materials. The 30 sentences from the Foss and Lynch (1969) materials used in Experiment 1 were also used in this experiment. Unlike Experiment 1, however, these sentences were presented in only one of two forms, regular or self-embedded unreduced. As in Experiment 1, 10 filler sentences were interspersed among the 30 target sentences, and the form in which the target sentences were presented was counterbalanced across subjects.

A two-alternative forced-choice recognition test for assessing memory of syntactic structure was constructed. This was done by simply deleting the self-embedded reduced alternatives from the surface structure recognition test used in Experiment 1.

Procedure. The procedure was very similar to that used in Experiment 1, differing in only three respects. First, in the acquisition phase, all sentences were presented for $12.5 \mathrm{sec}$. Second, the interpolated task between acquisition and testing consisted of rating several sets of words for category membership. This task took approximately the same amount of time as the personality attribute rating task used in Experiment 1. Third, on the recognition tests, subjects were instructed to rate how confident they were about each of their answers. They used a scale of 1 to 5 , with 1 indicating an absolute guess and 5 indicating complete certainty.

Subjects. Subjects were 22 students at the University of Colorado participating in partial fulfillment of an introductory psychology course requirement. One subject was replaced after postexperimental questioning indicated that he had expected a memory test and had attempted to learn the sentences.

\section{Results and Discussion}

The results paralleled those found in Experiment 1. For the word-choice task, there was no significant difference in the number of errors made due to sentence form (17\% error rate for regular sentences and $15 \%$ error rate for self-embedded sentences, $F<1$ ). There was a significant effect of sentence form for recognition performance $[\mathrm{F}(1,21)=10.18, \mathrm{MSe}=13.9, \mathrm{p}<.01]$, with more self-embedded sentences $(11.5$, on the average) correctly recognized than regular sentences ( 7.9 , on the average). Transforming these number-correct data into a proportion analogous to that used in Experiment 1 (number of items correctly recognized minus the number of items expected to be recognized by chance divided by the number representing perfect responding above that expected by chance) indicated that recognition performance in the present experiment was quite similar to that found in Experiment 1 . The average proportion of total possible correct responses above chance was .54 for self-embedded forms and .06 for regular forms in the present experiment, compared with .64 for self-embedded forms and .13 for regular forms in the first experiment (from first column of Table 3). Thus, these data are strong evidence that the superior memory shown for self-embedded forms in Experiment 1 was not an artifact of the post hoc scoring method used.

The confidence ratings were also analyzed. Average confidence ratings for four different types of responses were calculated. The four response types were correct self-embedded responses, correct regular responses, incorrect self-embedded responses, and incorrect regular responses. The mean confidence ratings for each of these response types is shown in Table 4.

A two-factor within-subjects analysis of variance was performed on these data (correctness of the response was one factor, and the sentence form selected was the other factor). Three subjects were not included in this analysis because they had no confidence ratings for incorrect regular responses, having made no incorrect regular responses. In general, subjects were more confident of correct responses than of incorrect responses $[F(1,18)=14.49$, MSe $=.26]$ and more confident of self-embedded responses than of regular responses $[F(1,18)=8.58, \mathrm{MSe}=.38]$. These main effects were qualified by a significant interaction $[F(1,18)=10.78$, $\mathrm{MSe}=.28]$. Inspection of Table 4 reveals that subjects reported being equally confident of their self-embedded

Table 4

Mean Confidence Ratings as a Function of Sentence Form and Correctness of Response

Correctness of Response

\begin{tabular}{lcc} 
Sentence Form & Correct & Incorrect \\
\hline Regular & 3.6 & 2.8 \\
Self-Embedded & 3.6 & 3.6 \\
\hline
\end{tabular}


responses and correct regular responses. Only on incorrect regular responses did subjects demonstrate a difference in the confidence of their responses, although even this difference was not very dramatic. Overall, then, subjects' confidence in their responses did not vary much.

\section{GENERAL DISCUSSION}

Both of the studies reported here are supportive of levels-of-processing type theories concerning material difficulty and retentivity. Consistent with Jacoby and Craik's (1969) ideas, the self-embedded sentences that were more difficult to process were better remembered than the more easily processed regular sentences, but only for memory of syntactic information. Memory for semantic information did not differ as a function of sentence type. These results, then, underscore the notion that the conclusions one draws concerning the memory effects of a variable such as material difficulty must take into account the relation between acquisition activities and the test task.

More specifically, these results, along with previous work (Kolers, 1973), provide evidence for the existence of an array of encoding operations, ranging from early graphemic analysis to higher level syntactic analysis, that can be involved in sentence processing and recognition. Typically, these operations will be performed in an automatic, routine manner, with more familiar stimuli requiring fewer of these operations. ${ }^{1}$ (Kolers, 1975; Lockhart et al., 1976). If, however, encoding at one of these levels is complicated (e.g., by inverting the typography or using unusual syntactic constructions), then additional, and perhaps more conscious, operations will need to be directed at that particular level in order to interpret the sentence. Furthermore, memory for the sentence will now include memory for that level of information requiring additional processing, even if this information is nonsemantic.

This view of sentence memory can be contrasted with the alternative view that the surface structure of a sentence is quickly discarded in memory, with only its meaning or deep structure being retained (Sachs, 1967). Past findings have argued against a strong version of the view that surface information is quickly forgotten. Tasks in which the subject is led to believe that surface information is important generally will promote memory for that surface information (e.g., Keenan, 1975; Toglia, 1976). A weaker statement of this theory, however, might hold that when extraction of meaning is the goal, surface information is quickly discarded. This interpretation does find support in the literature (Graesser \& Mandler, 1975; Sachs, 1967). The present results argue against even this weak statement. Surface information was remembered to some extent, even though the task involved extracting meaning as the primary objective. Apparently, if the material causes elaboration in surface domains, then that type of information will be remembered to some extent even if the task is to extract meaning. It is worth noting that there is another difference between the present study and studies that have not found memory for surface information after a semantic task. Other studies have tested for memory of the particular lexical items of the target sentence; the present results involve memory not for the particular lexical items, but for the manner in which the lexical items were arranged (syntactic form). This differing pattern of results indicates that the particular kind of surface information being tested may also influence whether memory for surface information will be demonstrated.

Many current theories maintain that surface or nonsemantic information will be remembered, but that it is quite susceptible to decay or interference (Craik \& Lockhart, 1972; Kintsch, 1975). Data from pilot work for the present research does attest to the dissipation of surface information. Recognition tests for surface structure were administered immediately after the acquisition phase, and recognition levels were nearly perfect. By contrast, the present study imposed a 10-min interval with an interpolated task between the completion of the acquisition phase and the administration of the recognition tests, and the levels of recognition for surface structure dropped substantially.

Another result of theoretical interest is the superior recognition of main clauses compared with subordinate clauses on the meaning test in Experiment 1. It is commonly found that information perceived to be important is remembered better than not-so-important information. Theoretically, the importance of an idea is marked by certain characteristic structures of a text or sentence. Kintsch (1975) has found that, for text, ideas delineated as superordinate (i.e., of primary importance) are remembered better than those ideas delineated as subordinate (i.e., elaborative). The present results parallel at the sentence level those findings at the text level. Ideas marked as important by the fact that they were contained in the main clause were better remembered than the ideas contained in the subordinate clause. This finding indicates that certain syntactic devices of sentences (e.g., clause position) do serve to characterize information as more or less important and, hence, will influence what information will be remembered.

Finally, this research would seem to have some pragmatic value with regard to instruction. A common strategy in an instructional setting is to design various tasks and exercises that facilitate assimilation of the to-be-learned material. Another strategy might be to present the materials in a form that itself would facilitate acquisition. Anderson (1976) has suggested that materials can be presented in a way that affords more elaborative processing than would otherwise be the case. Atkinson and Raugh (1975; Raugh \& Atkinson, 1975) have had success applying notions similar to Anderson's in a foreign language acquisition task. Based on the current results, a somewhat similar proposal might be made. Materials could be presented in 
a form that would induce elaborative processing, and hence better memory, for information in a desired domain.

\section{REFERENCES}

Anderson, J. R. Language, memory, and thought. Hillsdale, N.J: Erlbaum, 1976.

Atkinson, R. C., \& Raugh, M. R. An application of the mnemonic keyword method to the acquisition of a Russian vocabulary. Journal of Experimental Psychology: Human Learning and Memory, 1975, 104, 126-133.

Bransford, J. D., Franks, J. J., Morris, C. D., \& Stein, B. S. Some general constraints on learning and memory research. In L. S. Cermak \& F. I. M. Craik (Eds.), Levels of processing in human memory. Hillsdale, N.J: Erlbaum, 1979.

Bransford, J. D., \& McCarreld, N. S. A sketch of a cognitive approach to comprehension: Some thoughts about understanding what it means to comprehend. In W. B. Weiner \& D. S. Palermo (Eds.), Cognition and the symbolic processes. Hillsdale, N.J: Erlbaum, 1974.

Cooper, E. H., \& Pantle, A. J. The total-time hypothesis in verbal learning. Psychological Bulletin, 1967, 68, 221-234.

Craik, F. I. M., \& Lockhart, R. S. Levels of processing: A framework for memory research. Journal of Verbal Learning \& Verbal Behavior, 1972, 11, 671-684.

Fodor, J. A., \& GARRet, M. Some syntactic determinants of sentential complexity. Perception \& Psychophysics, 1967, 2, 289-296.

Foss, D. J., \& LyNCH, R. H., JR. Decision processes during sentence comprehension: Effects of surface structure on decision times. Perception \& Psychophysics, 1969, 5, 145-148.

Graesser, A., II, \& Mandlen, G. Recognition memory for the meaning and surface structure of sentences. Journal of Experimental Psychology: Human Learning and Memory, 1975, 104, 238-248.

Hakes, D. T., \& CaIRns, H. S. Sentence comprehension and relative pronouns. Perception \& Psychophysics, 1970, 8, 5-8.

Hakes, D. T., \& Foss, D. J. Decision processes during sentence comprehension: Effects of surface structure reconsidered. Perception \& Psychophysics, 1970, 8, 413-416.

JACOBY, L. L., \& CraIK, F. I. M. Effects of elaboration of processing at encoding and retrieval: Trace distinctiveness and recovery of initial context. In L. S. Cermak \& F. I. M. Craik (Eds.), Levels of processing in human memory. Hillsdale: N.J: Erlbaum, 1979.
KEENAN, J. M. The role of episodic information in the assessment of semantic memory representation for sentences. Unpublished doctoral dissertation, University of Colorado, 1975.

Kintsch, W. Memory representations of text. In R. L. Solso (Ed.), Information processing and cognition. Hillsdale, N.J: Erlbaum, 1975.

Kole Rs, P. A. Remembering operations. Memory \& Cognition, $1973,1,347-355$

Kole rs, P. A. Memorial consequences of automized encoding. Journal of Experimental Psychology: Human Learning and Memory, 1975, 1, 689-701.

Kolens, P. A. Reading a year later. Journal of Experimental Psychology: Human Learning and Memory, 1976, 2, 554-565.

Kolers, P. A., \& Ostry, D. J. Time course of loss of information regarding pattern analyzing operations. Journal of Verbal Learning and Verbal Behavior, 1974, 13, 599-612.

Lockhart, R. S., Craik, F. I. M., \& Jacoby, L. L. Depth of processing, recognition and recall. In J. Brown (Ed.), Recognition and recall. London: Wiley, 1976.

MASSON, M. E. J., \& SALA, L. S. Interactive processes in sentence comprehension and recognition. Cognitive Psychology, 1978, 10, 244-270.

McDaniel, M. A., Friedman, A., \& Bourne, L. E., Jr., Remembering the levels of information in words. Memory \& Cognition, 1978, 6, 156-164.

Raugh, M. R., \& Atrinson, R. C. A mnemonic method for the learning of a second language vocabulary. Journal of Educational Psychology, 1975, 67, 1-16.

SACHs, J. Recognition memory for syntactic and semantic aspects of connected discourse. Perception \& Psychophysics, 1967, 2 , 437-442.

Toglia, M. P. Verbatim and paraphrase memory for sentences. Unpublished doctoral dissertation, University of Colorado, 1976.

\section{NOTE}

1. This is not to imply that interpretation of a sentence involves a rigidly programmed sequence of analyses, but only that different kinds of information can, and will be, used for interpreting the sentence.

(Received for publication November 24, 1980; revision accepted March 5, 1981.) 